\title{
Preconception Healthcare: What Women Know and Believe
}

\author{
Keith A. Frey • Julia A. Files
}

Published online: 8 June 2006

(C) Springer Science+Business Media, Inc. 2006

\begin{abstract}
Objectives: The objectives of this study were to determine if women realize the importance of optimizing their health prior to a pregnancy, whether the pregnancy is planned or not; and to evaluate their knowledge level and beliefs about preconception healthcare. Additionally, we sought to understand how and when women wanted to receive information on preconception health. Methods: A survey study was performed using consecutive patients presenting to primary care practices for an annual wellwoman exam. Patients were recruited based on appointment type and willingness to complete the survey at the time of their appointment, but prior to being seen by the physician. Results: A total of 499 women completed the survey. Nearly all women $(98.6 \%)$ realized the importance of optimizing their health prior to a pregnancy, and realized the best time to receive information about preconception health is before conception. The vast majority of patients surveyed (95.3\%) preferred to receive information about preconception health from their primary care physician. Only $39 \%$ of women could recall their physician ever discussing this topic. The population studied revealed some significant knowledge deficiencies about factors that may threaten the health of mother or fetus. Conclusions: A majority of women do understand the importance of optimizing their health prior to conception, and look to their Primary care physician as their preferred source for such information. Study participants demonstrated deficiencies in their knowledge of risk factors that impact
\end{abstract}

K. A. Frey $(\bowtie) \cdot$ J. A. Files

Departments of Family Medicine and Women's Health Internal

Medicine, Mayo Clinic College of Medicine,

Mayo Clinic Arizona,

13737 N. 92nd Street, Scottsdale, AZ, 85260 USA

e-mail: frey.keith@mayo.edu maternal and fetal health suggesting that physicians are not addressing preconception healthcare during routine care.

Keywords Preconception care - Preconception health · Women's health $\cdot$ Primary care

\section{Introduction}

Preconception care is defined as the promotion of the health and well being of a woman and her partner before pregnancy [1]. The goal of the preconception visit is to identify medical and social conditions that may put the mother or fetus at risk. The concepts of preconception care have been articulated for over a decade - but unfortunately have not become part of the routine practice. Although many studies [2-9] document the effectiveness of interventions targeted to increase awareness of preconception folic acid supplementation, however, little evidence links comprehensive preconception health promotion to improved pregnancy outcomes. Only one study using data gathered more than 10 years ago demonstrated a greater likelihood of pregnancy intendedness in a low-income cohort of woman exposed to information on preconception health during routine family planning visits at a community health department [10]. Studies in the United Kingdom [11, 12] of knowledge and attitudes toward preconception care among primary health care teams show widespread consensus among the healthcare workers of the importance of the topic. One of these studies included the attitudes of women of childbearing age and noted that the view of the importance of preconception care was less strongly held by the female population studied [11]. Other studies have focused on more specific topics, such as rubella immunity, folic acid supplementation and glycemic control in diabetics [13-15]. Approximately $2 \%$ to $3 \%$ of all pregnancies result in a neonate 
with a serious genetic disease or a birth defect that can cause disabilities, mental retardation, and in some cases early death [16]. We are unaware of additional studies assessing the general knowledge and beliefs that women possess about optimizing their health prior to conception, or their preferences for obtaining such information.

\section{Methods}

A survey study of consecutive patients presenting to primary care practices for an annual well-woman exam was performed in accord with prevailing ethical principles. The selected primary care practices, occupying the same building at the Mayo Clinic Arizona, represented a women's health general internal medicine practice (5 physicians) and a family medicine practice ( 9 faculty physicians and 18 family medicine residents). Women were recruited for the study by nursing staff working within the practices, with permission and informed consent completed prior to distributing the survey tool. The Mayo Clinic IRB approved the study. Patients were recruited based on appointment type (e.g., well woman exam, annual Pap exam, and annual preventive medicine exam) and consenting to complete the survey questionnaire at the time of their appointment, but prior to being seen by the physician. The enrollment period was between August 2004 and July 2005. Women were considered eligible for the study if they were between the ages of 18 and 45 years, understood English, and gave their permission. The survey instrument was a four-page questionnaire and required approximately 10 minutes to complete. The survey included questions about demographics, pregnancy intendedness, knowledge and attitudes about preconception care, and personal preferences about sources of health information about preconception care.

Data from each survey was entered into a database at the Research Survey Center, Mayo Clinic, Rochester, Minnesota, and the aggregate data made available for analysis to the research team.

\section{Results}

A total of 570 women were invited to participate in the study; 58 declined and 13 did not meet eligibility criteria, leaving 499 women who completed surveys for data analysis. The demographic profile of the study population is shown in Table 1 . In this study population, the majority $(70.6 \%)$ of women were not currently attempting to conceive as noted in Table 2 . Approximately $5 \%$ were actively trying to conceive, with $13.5 \%$ and $8.4 \%$ considering a pregnancy in either the next 1-2 or 3-5 years respectively. Interestingly, of the women who had previously been pregnant, pregnancies had
Table 1 Study population demographic $(n=499)$

\begin{tabular}{lr}
\hline Age & \\
Range: 18 to 45 years & $24 \%$ \\
18 to 25 years & $30 \%$ \\
26 to 35 years & $46 \%$ \\
$\quad 36$ to 45 years & \\
Mean: 33 years & \\
Ethnicity & $84.8 \%$ \\
White & $3.6 \%$ \\
Asian & $1.3 \%$ \\
African-American & $1.4 \%$ \\
Native American & $9.0 \%$ \\
Other & \\
Education (highest level) & $0.4 \%$ \\
11 th grade or less & $7.5 \%$ \\
Graduated High School & $33.0 \%$ \\
Some college or technical school & $38.5 \%$ \\
Graduated college & $6.7 \%$ \\
Some graduate work & $14.0 \%$ \\
Graduate degree & \\
Household income & $10.5 \%$ \\
Less than $\$ 25,000$ & $19.7 \%$ \\
\$26,000 to $\$ 50,000$ & $18.6 \%$ \\
\$51,000 to $\$ 75,000$ & $13.0 \%$ \\
\$76,000 to $\$ 99,000$ & $13.2 \%$ \\
\$100,000 to $\$ 125,000$ & $6.8 \%$ \\
\$126,000 to $\$ 150,000$ & $6.4 \%$ \\
\$151,000 to $\$ 200,000$ & $11.8 \%$ \\
Greater than $\$ 200,000$ & \\
\hline
\end{tabular}

actually been planned in only $47.2 \%$ of instances. Nearly all women in the study $(98.6 \%)$ realized the importance of optimizing their health prior to a pregnancy, again as noted in Table 2 . However, only 39\% could ever recall their physician discussing preconception health. The majority of the women in this study population who were interested in preconception health education preferred the information prior to a pregnancy $(74.8 \%)$ or at the time of their annual medical exam (11.9\%), as displayed in Fig. 1.

The women who were interested in preconception health education, or unsure of such interest, were asked their preferences for sources for such information (Table 3 and Fig. 1). The vast majority preferred their physician, either a primary care physician $(51.3 \%)$ or Obstetrician/gynecologist $(44.0 \%)$. Only a fraction would primarily seek their information from sources other than their physician.

The survey included questions about patient awareness of key preconception risk factors which may influence the outcome of a future pregnancy. The study population demonstrated high awareness of certain risk factors, such as tobacco, alcohol, drug use, and domestic abuse (Table 4). There were, however, opportunities for improvement in basic understanding of the risk to maternal/fetal health as it relates to fish consumption, exposure to cat litter, and the impact of family and/or genetic history. 
Table 2 Conception history and planning

\begin{tabular}{|c|c|}
\hline \multicolumn{2}{|l|}{ Plans about getting pregnant } \\
\hline No plans at present time & $70.6 \%$ \\
\hline Currently trying & $4.6 \%$ \\
\hline Considering in next 1 to 2 years & $13.5 \%$ \\
\hline Considering in next 3 to 5 years & $8.4 \%$ \\
\hline Have tried, unable to get pregnant & $2.8 \%$ \\
\hline \multicolumn{2}{|l|}{ Ever been pregnant? } \\
\hline Yes & $50.7 \%$ \\
\hline No & $49.3 \%$ \\
\hline \multicolumn{2}{|c|}{ If ever pregnant, where previous pregnancies planned? } \\
\hline Yes & $47.2 \%$ \\
\hline No & $52.8 \%$ \\
\hline \multicolumn{2}{|c|}{ Affect of optimizing health of mother and pregnancy? } \\
\hline Has a good effect on the pregnancy & $98.6 \%$ \\
\hline Has no effect on the pregnancy & $0.8 \%$ \\
\hline Has a bad effect on the pregnancy & $0.6 \%$ \\
\hline \multicolumn{2}{|c|}{ Doctor ever spoken to you about preconception health? } \\
\hline Yes & $39.0 \%$ \\
\hline No & $61.0 \%$ \\
\hline \multicolumn{2}{|c|}{$\begin{array}{l}\text { Are you interested in receiving preconception health } \\
\text { education? }\end{array}$} \\
\hline Very interested & $34.8 \%$ \\
\hline Somewhat interested & $21.6 \%$ \\
\hline Unsure & $10.1 \%$ \\
\hline Not at all interested & $33.5 \%$ \\
\hline \multicolumn{2}{|c|}{$\begin{array}{l}\text { If interested or unsure about education, when would you } \\
\text { prefer? }\end{array}$} \\
\hline At the time I become pregnant & $7.6 \%$ \\
\hline Before I try to get pregnant & $74.8 \%$ \\
\hline During pregnancy & $0.7 \%$ \\
\hline Every time I get an annual medical exam & $11.9 \%$ \\
\hline Unsure & $5.0 \%$ \\
\hline
\end{tabular}

Table 3 Patient preferences for sources of preconception information (Percentage ranking the choice as first preference)

\begin{tabular}{lr}
\hline Primary care physician & $51.3 \%$ \\
Obstetrician/gynecologist & $44.0 \%$ \\
Family, friends & $0.3 \%$ \\
Magazine, newspaper & $0.3 \%$ \\
Internet/world wide web & $3.1 \%$ \\
Other & $0.7 \%$ \\
\hline
\end{tabular}

\section{Discussion}

Preconception care is the primary prevention of maternal and perinatal morbidity and mortality. It is an important issue in women's health that is easily overlooked by physicians and patients. The results of this study demonstrate that the vast majority of study participants understood the importance of preconception healthcare and realized that it should be obtained prior to conception. Interestingly, many of the women who expressed an interest in receiving information about preconception healthcare perceived the annual exam as the appropriate venue for exploring this topic. The study participants showed a strong preference for obtaining information about preconception healthcare from their personal physician and the majority eschewed the use of technology such as the Internet as a source of such information. The study revealed that a large percentage of women expected their primary care physician or OB/GYN physician to address preconception healthcare with them.

These data demonstrate that the study population exhibited gaps in knowledge about specific preconception health topics, but confirmed previous findings that women do have

Fig. 1 Are you interested in preconception health education?

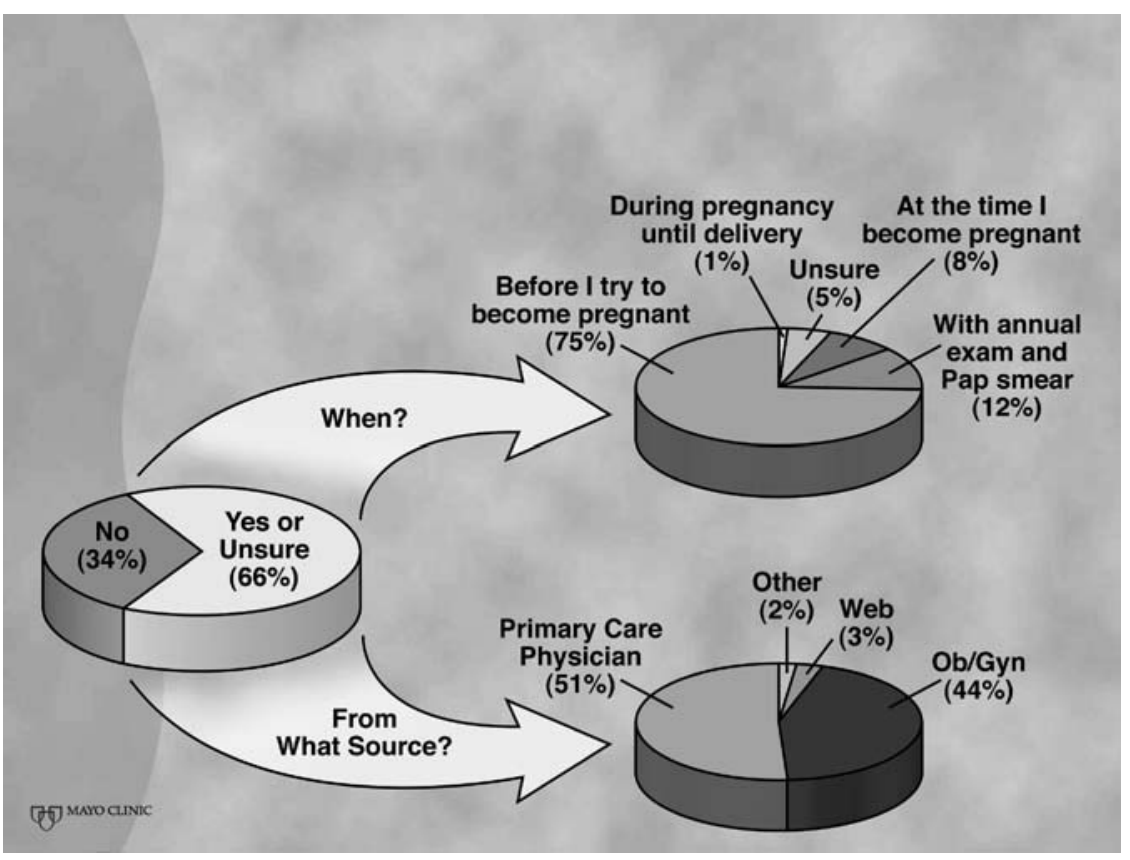


Table 4 Preconception health knowledge and opinions (Percentage aware $^{\mathrm{a}}$ of risk factor potentially affecting a pregnancy)

\begin{tabular}{ll}
\hline Consumption of certain fish & $54.2 \%$ \\
Exposure to cat liter & $64.9 \%$ \\
Folic acid use & $79.6 \%$ \\
Impact of family and/or genetic history & $84.1 \%$ \\
Infectious diseases (need to screen for) & $89.3 \%$ \\
Immunizations (up to date) & $91.2 \%$ \\
Alcohol use & $95.8 \%$ \\
Abuse (verbal, sexual and/or physical) & $97.2 \%$ \\
Medication use (prescription and nonprescription) & $97.4 \%$ \\
Tobacco use & $98.2 \%$ \\
Illicit drug use & $98.8 \%$
\end{tabular}

${ }^{\mathrm{a}}$ Agreed or strongly agreed on a five point Likert scale

an increased awareness of the importance of folic acid supplementation [2-9]. Additionally, the study population showed a high awareness of the risks associated with tobacco, alcohol and drug use, but were much less aware of the risks to fetal health associated with fish consumption and exposure to cat litter. Such findings point out the need to continue efforts at increasing public awareness of other modifiable risks to fetal health. Unfortunately, fewer than $40 \%$ of the women surveyed recalled discussing preconception healthcare with their physician at the time of their annual exam.

Clearly, there is a gap in patient expectation and delivery of healthcare services in this population of educated middle class women. This study only surveyed women who had chosen to obtain their primary care in Internal Medicine and Family Medicine practices of a private health care facility, suggesting that there may be obstacles to delivering preconception care even in an academic outpatient setting. Potential explanations for this gap in the delivery of preconception healthcare may include: age of patients studied, time constraints in the outpatient setting or insufficient training and/or content knowledge in preconception healthcare of the physicians. This study demonstrates that an opportunity to better understand the gap in care delivery and to evaluate possible solutions in private middle class settings. More exposure on the part of residents to a curriculum on preconception healthcare may provide an opportunity for physicians in these specialty fields to improve their skills and the delivery of this much-needed care to women.

One of the limitations of our study was the homogeneity of our patient population. The majority of our study participants were middle class, Caucasian and had at least some college education. Our findings may not be broadly applicable to women of other socioeconomic backgrounds, ethnicity and educational levels. The lack of diversity of our population reflects the demographics of this community and further study in other populations should be pursued.

As all women of reproductive age and potential presenting for continuing care in the primary care setting are candidates for preconception care, the essential and critical role of primary care physicians and providers in the provision of preconception care is apparent. The Centers for Disease Control (CDC) has published their "Recommendations for Improving Preconception Health and Health Care" [17]. These recommendations were developed through a collaborative effort during which the CDC successfully aligned the missions of a number of its external partners and internal programs to ultimately draft these national recommendations for preconception care. These national recommendations can now serve as a roadmap for both graduate medical education and continuing medical education curricula to improve the knowledge and skill of the physician workforce in the delivery of comprehensive preconception health care.

Acknowledgments The authors express their appreciation to Joseph Hentz and Brie Noble for assisting in the data handling and analysis, the nursing staff in the practices of Women's Health Internal Medicine and Family Medicine at the Mayo Clinic Thunderbird site for patient recruitment, and Dr Paru David for her assistance in the survey design. A grant from the Mayo Clinic Arizona Research Committee supported the non-personnel costs associated with this study.

\section{References}

1. Frey K. Preconception care by the nonobstetrical provider. Mayo Clin Proc 2002;77:469-73.

2. Morin VI, Mondor M, Wilson RD. Knowledge on periconceptional use of folic acid in women of British Columbia. Fetal Diagnos Ther 2001;16:111-5.

3. Morin P, De Wals P, St-Cyr-Tribble D, Niyonsenga T, Payette H. Pregnancy planning: a determinant of folic acid supplements use for the primary prevention of neural tube defects [see comment]. Can J Public Health. Revue Canadienne de Sante Publique 2002;93:259-63.

4. Sayers GM, Hughes N, Scallan E, Johnson Z. A survey of knowledge and use of folic acid among women of child-bearing age in Dublin. J Public Health Med 1997;19:328-32.

5. Watson M, Watson L, Bell R, Halliday J. The increasing knowledge of the role of periconceptional folate in Victorian women of childbearing age: follow-up of a randomised community intervention trial. Aust N Z J Public Health 2001;25:389-95.

6. de Jong-Van den Berg LT, Hernandez-Diaz S, Werler MM, Louik $\mathrm{C}$, Mitchell AA. Trends and predictors of folic acid awareness and periconceptional use in pregnant women. Am J Obstet Gynecol 2005;192:121-8

7. Chacko MR, Anding R, Kozinetz CA, Grover JL, Smith PB. Neural tube defects: knowledge and preconceptional prevention practices in minority young women. Pediatrics $2003 ; 112: 536-42$.

8. Perlow JH. Comparative use and knowledge of preconceptional folic acid among Spanish- and English-speaking patient populations in Phoenix and Yuma, Arizona. Am J Obstet Gynecol 2001;184:1263-6.

9. Brandenburg H, Traas MA, Laudy J, Ursem N, Westerveld AM, Wladimiroff JW. Periconceptional use of folic acid amongst women of advanced maternal age.[see comment]. Prenatal Diagnos 1999;19:132-5.

10. Moos MK, Bangdiwala SI, Meibohm AR, Cefalo RC. The impact of a preconceptional health promotion program on intendedness of pregnancy. Am J Perinatology 1996;13:103-8. 
11. Wallace M, Hurwitz B. Preconception care: who needs it, who wants it, and how should it be provided?[see comment]. Br J Gen Pract 1998;48:963-6.

12. Heyes T, Long S, Mathers N. Preconception care: practice and beliefs of primary care workers. Fam Pract 2004;21:22-7.

13. Farrell M. Improving the care of women with gestational diabetes. MCN, Am J Matern Child Nurs 2003;28:301-5.

14. Bernasko J. Contemporary management of type 1 diabetes mellitus in pregnancy. Obstet Gynecol Surv 2004;59:628-36.
15. Holing E. Preconception care of women with diabetes: the unrevealed obstacles. J Matern Fetal Med 2000;9:10-3.

16. Ensenauer R, Reinke S, Ackerman M, Tester D, Whiteman D, Tefferi A. Primer on medical genomics Part VIII: essentials of medical genetics for the practicing physician. Mayo Clin Proc 2003;78:846-57.

17. Centers for Disease Control. Recommendations for improving preconception health and health care. MMWR, April 20, 2006. 